\title{
La main invisible suffit-elle pour assurer le développement des territoires? Évolution récente des politiques gouvernementales en matière de développement régional
}

\author{
Jean-François Simard, Ph. D. \\ Professeur-chercheur à l'Université du Québec en Outaouais \\ Yvon Leclerc, Ph. D. \\ Professeur-chercheur en développement culturel et territorial
}

\section{INTRODUCTION}

Au moment d'écrire ces lignes, les politiques et les programmes de développement régional mis en place au cours des 30 dernières années connaissent un bouleversement sans précédent qui met fin à ce qu'il est convenu d'appeler « le modèle québécois de développement territorial ». Jamais n'a-t-on vu une remise en question aussi radicale des dispositifs mis en place depuis la Révolution tranquille afin de soutenir le développement local et régional. Un modèle qui pourtant avait fait ses preuves en matière de création d'emplois et de croissance de la richesse collective.

Quand on sait que la mise en place des conditions favorables au développement local exige de longues années d'apprivoisement des acteurs et des institutions locales, il y a de quoi s'inquiéter. La précipitation avec laquelle ces changements surviennent, sans analyse sérieuse, sème le doute sur les intentions réelles qui se cachent derrière cette grande braderie politico-administrative. Quels sont ces principaux changements et quels enjeux se cachent derrière ce saccage des structures administratives territoriales que sont plus particulièrement les CLD (Centre local de développement), les CRÉ (Conférence régionale des élus) et les organismes jeunesse?

Un bouleversement sans précédent qui met fin à ce qu'il est convenu d'appeler « le modèle québécois de développement territorial. »

\section{LE TERRITOIRE AU REGARD DE L'ÉTAT : AU-DELÀ DU FORDISME}

Le président fondateur de la Fondation de l'entrepreneurship, Paul-Arthur Fortin, a toujours soutenu que chaque milieu disposait des moyens d'assumer son développement (Fortin, 2002). Cette affirmation, largement partagée, se fondait sur les connaissances du système économique dont on disposait lors de la création de cette institution, en 1980. Or, trente-cinq ans plus tard, en regard des nouvelles théories sur le développement endogène (Guellec et Ralle, 2003), ce postulat ne résiste plus à l'analyse, car ces connaissances évaluaient le système économique de la période fordiste: un modèle de concertation économique basé sur la trilogie État-Syndicat-Patronat.

À la fin des années 1980, les travaux des chercheurs révélaient l'importance des disparités régionales au
Québec. Grosso modo, les indicateurs socioéconomiques montraient un appauvrissement des centres-villes (l'effet trou de beigne), un enrichissement de la couronne des villes et un appauvrissement à partir de la deuxième couronne et du reste du territoire. La recension des écrits indique une prise de conscience semblable dans plusieurs pays occidentaux.

Dans l'ordre économique et malgré des progrès évidents, l'État fordien s'est révélé incapable de corriger les problèmes soulevés par un «maldéveloppement» qui a conduit la population de certains quartiers urbains anciens à basculer dans la dépendance des programmes sociaux comme l'a bien démontré le Conseil des affaires sociales, en 1989, avec les données des recensements des 
années 1970 et 1980 . Cette situation s'est aggravée dans une économie postfordiste. Sur tous les continents, les instances de recherche internationales, et en particulier l'Organisation pour la coopération et le développement économique (OCDE), constatent que les États qui abdiquent devant les forces du marché s'exposent à un accroissement des écarts entre les riches et les pauvres. La Main invisible ne suffit pas à réduire les disparités régionales; bien au contraire, elle les aggrave au profit des grandes métropoles.

\section{La Main invisible ne suffit pas à réduire les disparités régionales; bien au contraire, elle les aggrave au profit des grandes métropoles.}

\section{DE LA MONTÉE DE LA SOCIÉTÉ CIVILE DANS LE DÉVELOPPEMENT LOCAL}

C'est toutefois dans l'ordre social que les changements les plus significatifs se remarquent durant cette période. Le modèle de développement à trois pôles (État, marché et syndicats) de la société fordiste s'est enrichi d'un quatrième pôle, celui de la société civile formée d'hommes et de femmes, souvent bardés de diplômes, capables de se faire entendre (Fillion, 1995; Fontan, Lévesque et Klein, 2003). Dans l'économie postfordiste, la société civile ne se contente plus de contester l'ordre établi, comme à la fin des années 1960 et durant les années 1970; désormais, elle innove en entreprenant des projets de développement local. Elle se pose même en alliée de l'État dans sa définition des conditions requises pour la mise en place de services de proximité qui contribuent à la satisfaction des besoins de la population.

Dès le début des années 1980, les différents paliers de gouvernements ont intégré la société civile à leur logique de développement. On parle dès lors de « gouvernance territoriale ». C'est le gouvernement fédéral qui, le premier, donnera le ton. Il entreprendra de répondre aux besoins des régions rurales en favorisant la mobilisation des forces locales et en appuyant financièrement le démarrage d'entreprises. Ainsi sont nés les Centres d'aide au développement des collectivités (CADC) et les Centres d'aide aux entreprises (CAE). Entièrement financées par Ottawa, les deux structures ont fusionné au début des années 1990 et sont devenues les Sociétés d'aide au développement des communautés (SADC). Un nouveau modèle de développement était né.

L'alliance État/communauté locale proposait un dépassement des traditionnelles logiques endo- gènes de développement qui, plus souvent qu'autrement, ne conduisait qu'à la répétition des processus d'appauvrissement et à la perpétuation des conditions ne permettant pas le décollage économique (théories dites du take-off). Ces changements étaient confirmés, au milieu des années 1980, par les recherches menées par le Groupe de recherche européen sur les milieux innovateurs (GREMI), qui démontraient que l'innovation n'était plus l'apanage de la grande entreprise, comme à l'époque fordiste des Trente glorieuses, mais dans les milieux où la population se mobilisait pour développer des projets (Aydalot, 1986).

Avec cette nouvelle forme d'alliance fédérale-provinciale-municipale-société civile, nous assistions à l'émergence d'une forme d'interscalarité du développement territorial.

C'est aussi au milieu des années 1980, dans les quartiers pauvres de Montréal, que sont nées les Corporations de développement économique communautaire (CDEC) qui avaient également pour mission de rassembler les forces du milieu. Le gouvernement du Québec est le principal bailleur de fonds des CDEC qui sont aussi financées par le gouvernement fédéral et dans une moindre mesure, par la Ville de Montréal (Malo et Moreau, 1999). Avec cette nouvelle forme d'alliance fédérale-provinciale-municipale-société civile, nous assistions à l'émergence d'une forme d'interscalarité du développement territorial (jeu d'échelles favorisant un développement intégré), confirmant ainsi que le développement n'est pas que le résultat d'un mouvement descendant (top 
down) ou ascendant (bottom up), mais un maillage des deux.

Puis, au milieu des années 1990, le gouvernement du Québec décidait la mise sur pied de deux organisations; l'une pour la concertation régionale les Conseils régionaux de développement (CRD) et l'autre pour le développement local - les Centres locaux de développement (CLD), toutes deux constituées par l'Assemblée nationale en décembre 1997. Nous étions alors en présence du modèle de décentralisation administrative le plus achevé en

\section{DE LA DILUTION DE LA SOCIÉTÉ CIVILE}

Le nouveau gouvernement élu en 2003 à Québec voyait les choses autrement. En toute légitimité et de bonne foi, il confiait aux élus locaux la responsabilité du développement local et levait l'interdit pour un groupe d'occuper plus de $50 \%$ des sièges du conseil d'administration du CLD et de la Conférence régionale des élus qu'il créait en lieu et place des Conseils régionaux de développement. Nous avons déjà qualifié, dans un article antérieur, ce choix de la «municipalisation » du développement local et régional (Simard et Leclerc, 2008). Déjà, à partir de 2004, cette décision renvoyait chez eux plusieurs centaines de bénévoles qui siégeaient à ces conseils d'administration et qui souhaitaient s'investir dans le développement de leur communauté. Comme si les élus municipaux pouvaient se priver de leur compétence dans le développement de leur milieu.

$\mathrm{Au}$ final, le comportement actuel du gouvernement met en bière les deux manières éprouvées jusqu'ici pour rétablir un certain équilibre entre territoires favorisés et défavorisés : il coupe les vivres accordées aux MRC et congédie les bénévoles qui s'activent dans le développement local au sein des CLD. En outre, les compressions financières demandées à Solidarité rurale et au

matière de développement territorial à voir le jour dans toute l'histoire de l'administration publique québécoise.

Toutes ces organisations avaient deux objectifs communs : mobiliser les forces de leur milieu et soutenir financièrement la création d'entreprises. Ces choix structurels répondaient en tout point à l'évolution de notre système économique qui accorde une place à la participation citoyenne et à l'économie sociale comme une autre façon d'aborder le développement économique.

programme de soutien financier aux municipalités dévitalisées laissent croire que le développement local peut s'accomplir par la Main invisible, sans le support de l'État.

Or, cette question, bien documentée par la recherche et les statistiques, démontre la nécessité de l'intervention de l'État pour lutter contre les inégalités. Fort heureusement, certaines MRC ont choisi de conserver leur CLD, même si leurs budgets ont été réduits de $55 \%$.

Par ces décisions, le gouvernement du Québec touche les deux fonctions qu'il s'était données pour minimiser l'exode des cerveaux vers les villes et réduire les écarts de développement entre les territoires : favoriser la mobilisation des acteurs et soutenir financièrement l'entrepreneuriat local.

Heureusement, les régions rurales peuvent encore compter sur les SADC soutenues financièrement par le gouvernement fédéral. Incidemment, la plupart des SADC se sentiront bien orphelines, car elles avaient développé une collaboration active avec les CLD dans le montage financier de nombreux projets.

En somme, les décisions du gouvernement font poindre la crainte de trois types d'appauvrissement :

- Un appauvrissement politique. L'évacuation de la présence du gouvernement du Québec en matière de développement local, alors que dans les faits, cette compétence était devenue avec le temps, presque exclusivement de juridiction provinciale. L'État du Québec perd ainsi une capacité d'agir sur le développement de son 
propre territoire, laissant totalement aux SADC le mandat de le faire. Pour la plus grande province du Canada, ce retrait n'est pas banal... Qui plus est, cela accrédite le retour du scénario d'un État québécois unitaire, au sein duquel la question de la décentralisation est un encombrement administratif dont on ne peut se payer le luxe en période de redressement des finances publiques. Pour l'heure, rien n'indique que le transfert de la compétence du développement économique aux municipalités lors du pacte fiscal actuellement en préparation viendrait combler le retrait de l'intervention de l'État dans le développement local et la lutte aux disparités régionales.

- Un appauvrissement économique. La perte de l'expertise en matière de développement local et régional qui aura pris une décennie à s'édifier demeure difficilement quantifiable, surtout à court terme. Toujours est-il que nous assistons à un démembrement de l'administration publique régionale et supra municipale qui ne peut pas ne pas occasionner une altération significative du capital de développement économique local et régional. On assiste à une individualisation du développement et à une évacuation de la

\section{L'OCCASION D'ALLER PLUS LOIN?}

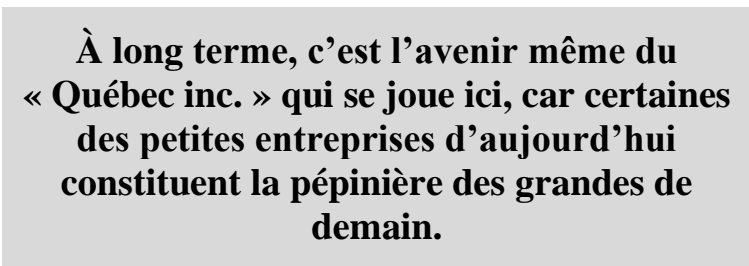

Les porte-parole du gouvernement et certains élus municipaux répètent que la grande braderie en cours offre l'occasion d'améliorer le modèle existant. Fallait-il pour ce faire jeter le bébé avec l'eau du bain? Le modèle actuel avait sans doute ses failles qui devaient être corrigées. Nous avons déjà identifié dans une recherche antérieure qu'au regard de la reddition de compte, les CLD éprouvaient d'importantes lacunes; de même, l'évaluation de la performance demeurait déficiente (Simard et Leclerc, 2012). Par ailleurs, la démocratisation des mécanismes de développement devait être consolidée et la nécessité d'aller plus loin dans le processus de décentralisation et d'imputabilité solidarité entre les acteurs. C'est toute la capacité d'action du développement économique locale qui souffre présentement de l'absence des structures de concertation régionale et de développement local. Du reste, on ne sait pas encore sous quelle forme pourrait renaitre la productive alliance État-communauté locale instituée progressivement depuis la Révolution tranquille.

- Un appauvrissement social. Le démembrement complet d'un dispositif de développement (nous parlons ici de la fin d'un modèle, insistons sur ce point) et cela, sans véritables débats politiques et sans aucun mandat populaire pour le faire. Pour la première fois de notre histoire nationale, nous nous retrouvons sans aucune forme d'instances régionales avec lesquelles l'État pourra dialoguer - même du temps de la colonisation l'État central était en lien avec les diocèses (Harvey, 2002) - et au sein desquelles les collectivités supra municipales pouvaient se concerter. Laissées à elles-mêmes, privées des dispositifs qui avaient pour mission de relancer le développement des territoires, les MRC réussiront-elles à maintenir le rythme de la dernière décennie?

constituait toujours un enjeu politique important dont il fallait discuter.

Mais pour autant, la nécessité de rafraîchir le modèle québécois en matière de développement territorial n'exigeait pas son abolition pure et simple. Rien en termes de rationalité économique ou politique ne le justifiait. Nous n'avions pas besoin d'une telle braderie pour atteindre le nécessaire équilibre des finances publiques. Le gouvernement Couillard propose un « grand bond en arrière », vers un style de gouvernance qui repose sur une dynamique de centralisation du pouvoir, accompagné d'un laisser-faire qui, à terme, ne pourra pas ne pas avoir de conséquences sur l'entrepreneurship québécois et l'enracinement territorial du capital économique du Québec. À long terme, c'est l'avenir même du «Québec inc. » qui se joue ici, car certaines des petites entreprises d'aujourd'hui constituent la pépinière des grandes de demain. 
Jusqu'ici, l'expérience québécoise et internationale de même que la recension des écrits (les projets Leader de l'Union européenne, par ex.) montrent que le redéploiement des territoires requiert l'intervention de l'État central sous deux formes : favoriser la mobilisation des acteurs et accorder un appui financier aux initiatives entrepreneuriales. Cette formule d'intervention offre l'avantage de respecter les choix des acteurs locaux et de les mobiliser dans leur mise en œuvre.

\section{CONCLUSION PROVISOIRE}

\section{Derrière le choix du gouvernement \\ Couillard d'abolir le modèle québécois en \\ matière de développement local et régional se cachent deux visions totalement opposées de la gouvernance territoriale et plus globalement de l'avenir du Québec.}

Devant autant d'improvisation, il est difficile d'écrire autre chose qu'une conclusion provisoire... Nous pouvons néanmoins tabler sur une grande certitude. Derrière le choix du gouvernement Couillard d'abolir le modèle québécois en matière de développement local et régional se cachent deux visions totalement opposées de la gouvernance territoriale et plus globalement de l'avenir du Québec. Disons-le clairement : nous sommes en face d'un choix de société.

Il y a d'une part ceux qui ont une confiance absolue dans les seules forces du marché pour assurer le développement territorial du Québec et qui s'imaginent qu'un État super centralisé aura la sensibilité requise pour venir corriger ponctuellement les défiances de cette logique. C'est un retour à un État régalien que même Adam Smith n'avait pas imaginé.

Il y a d'autre part ceux qui s'appuyant sur le dynamisme du marché, croient que le capital social n'est pas toujours au rendez-vous afin de soutenir adéquatement le développement de l'entrepreneurship et que dans ce cas, le soutien de l'État, par le biais d'une administration publique décentralisée, est plus à même de répondre aux besoins du milieu.
Le gouvernement aura beau dire et répéter que les régions, les villes de plus de 100000 habitants et les MRC doivent choisir leur propre mécanisme de développement, personne n'est dupe. Il devra, grosso modo, revenir au modèle qui a été mis en place au fil des ans et qui est arrimé à l'évolution de l'économie. D'abord, créer un dispositif qui favorisera le regroupement et la mobilisation des forces de chaque milieu, et ensuite lui accorder une aide financière modulée selon un indice du développement de chaque milieu. La roue existe déjà, monsieur Couillard!

Aujourd'hui, il revient légitimement au gouvernement Couillard de proposer son modèle de développement pour le Québec; demain, il reviendra aux Québécois et aux Québécoises d'en disposer en exerçant leur droit démocratique.

\section{BIBLIOGRAPHIE SOMMAIRE}

Aydalot, P. (1986). Milieux innovateurs en Europe, Paris : Économica/GREMI.

Filion, P. (1995). «Urbanisation et transition économique : du fordisme à l'après-fordisme ». Dans Gagnon, A. G. et A. Noël, L'espace québécois, Montréal, Les Éditions Québec/Amérique, coll. «Société Dossiers/ documents » : 189-215.

Fontan, J.M., Klein, J.L. et B. Lévesque. (dir.) (2003). Reconversion économique et développement territorial, Sainte-Foy, Presses de l'Université du Québec.

Fortin, P. A., \& Fondation de l'entrepreneurship. (2002). La culture entrepreneuriale, un antidote à la pauvreté. Éditions Transcontinental.

Guellec, D. et Ralle, P. (2003) Les nouvelles théories de la croissance, Collection repères, Éditions La Découverte.

Harvey, F. (2002) «Le diocèse catholique au Québec: un cadre territorial pour l'histoire sociale » in Les Cahiers des dix, Numéro 56, pp. 51-124.

Malo, M. C., et Moreau, C. (1999). «Corporations de développement économique communautaire (CDÉC) et Caisses Desjardins : quelle configuration partenariale? » in Lien social et Politiques, (41), pp. 119-128.

Simard, J.F. et Leclerc, Y. (2008). « Les Centres locaux de développement 1998-2008. Une gouvernance en mutation: entre participation citoyenne et imputabilité municipale». In Revue canadienne des sciences régionales, volume 31, numéro 3, pp. : 615-636.

Simard, J.F. et Leclerc, Y (2012) «La reddition de compte: le maillon faible du développement local? Regards sur les centres locaux de développement » in Économie et Solidarités, Volume 42, numéro 12, pp. 105-127. 


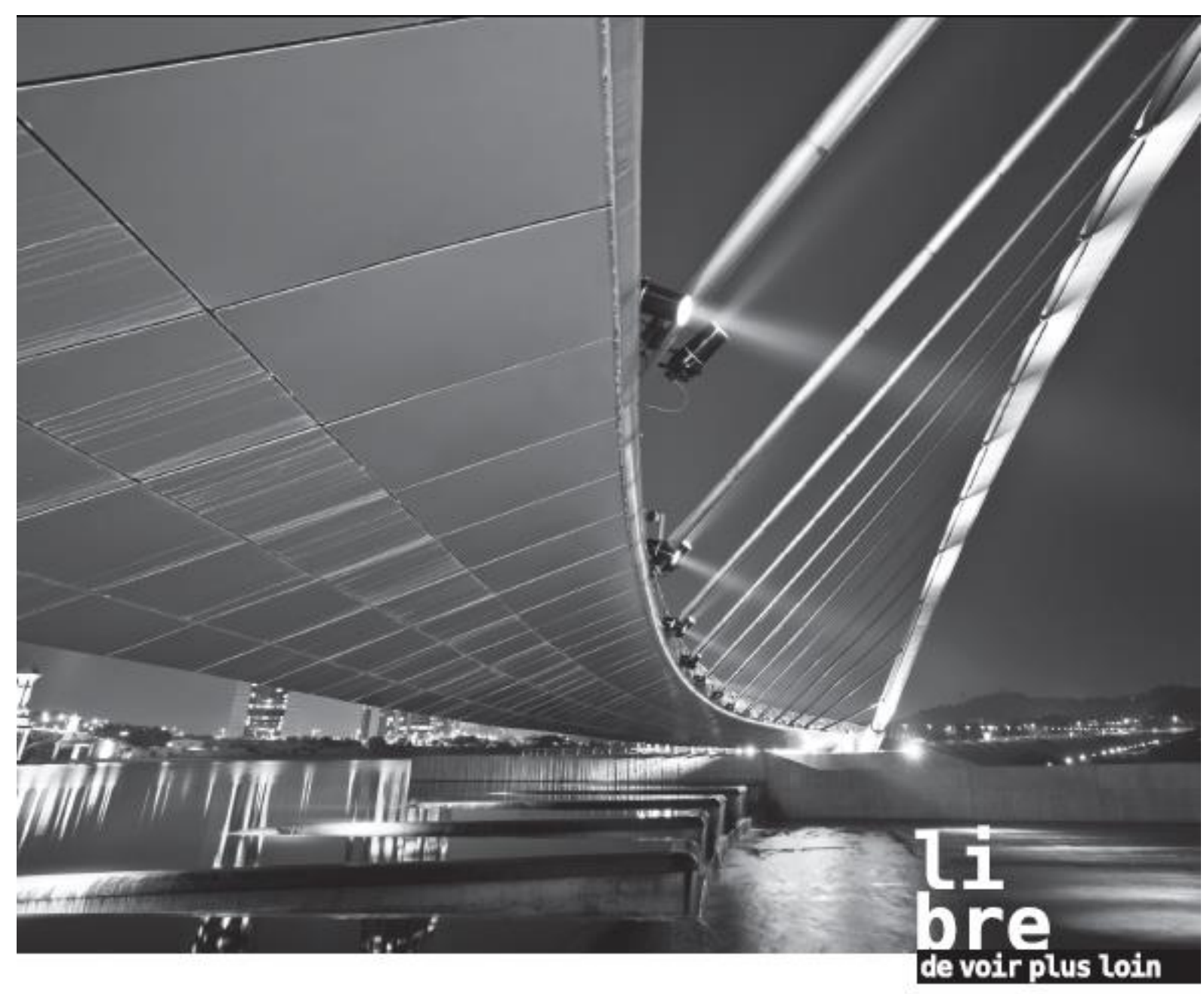

\section{DOCTORAT}

\section{EN MANAGEMENT DE PROJETS}

- Programme novateur et unique dans la francophonie

- Bourses du fonds de soutien du doctorat en management de projets

Université du Québec à Chicoutimi

418 545-5011, poste 5282

uqac.ca/dmp

uqac.ca

UQAC 